\title{
Exercise into Size
}

\section{Poetry}

The fact about exercise

Must not be at any size

Intense enough for sweat

Rate of heart must maximize

Should exercise every day

Circadian knows the way

Eating resting need drop

For the balance of the pay

No nutrition does the job

Small enough keep the gob

Evening becomes crucial

When insulin comes to fob

To tolerate glucose

You must use up adipose

Burning fatty of belly

You must not laze overdose

Listen but not overlook,

This is tip of multi-book

Move and move and exercise,

Not be trapped by the hook. ${ }^{1-10}$

\section{Acknowledgements}

None.

\section{Conflict of interest}

Author declares that there is no conflict of interest.
Special Issue - 2015

\author{
Akbar Nikkhah \\ Department of Animal Sciences, University of Zanjan, Iran
}

Correspondence: Akbar Nikkhah, Chief Highly Distinguished Professor, Department of Animal Sciences, Faculty of Agricultural Sciences, University of Zanjan, Foremost Principal Highly Distinguished Elite-Generating Scientist, National Elite Foundation, Iran, Email anikkha@yahoo.com

Received: October 03, 2015 | Published: October 5, 2015

\section{References}

1. Nikkhah A. A Time-Efficient Exercise Formula for Normalizing Obese Body Mass Index. Adv Obes Weight Manag Control. 2015;3(2):00049.

2. Nikkhah A. Diabesity and Lifestyle. Curr Res Diabetes Obes J. 2015;1(1):CRDOJ.MS.ID.555552.

3. Nikkhah A. A Realistic Weight Management Nutritional Regimen for Today's Life. J Nutr Health Food Eng. 2015.

4. Nikkhah A. Lifestyle Optimization: Today's Foremost Probiotic. J Probiotics Health. 2015;3(2):e119.

5. Nikkhah A. Scheduling Exercise-Eating to Optimize Gut Probiotics: A Global Invention. J Probiotic Health. 2015;3:e118.

6. Nikkhah A. Establishing Regular Patterns of Cellular Mechanics to Minimize Oncogenesis: Animal Sciences Inspire. Aust J Vet Sci Anim Husb. 2015;2(2):1010.

7. Nikkhah A. Slowing Aging via Joint Rhythmic Exercise and Optimized Eating Behavior: Nature Enthuses. J Bioprocess Biotech. 2015;5:e136.

8. Nikkhah A. Towards a Global Anti-Diabetes Exercise Program. $J$ Bioprocess Biotechniq. 2015;5:e135.

9. Nikkhah A. Matching Substrate Provision and use to Power Cut Oncogenesis. J Cancer Prev Curr Res. 2015;3(2):71.

10. Nikkhah A. Circadian Timing and Regularity of Physical Activity: A Novel Bioprocess to Prevent Devastating Modern Diseases. J Bioprocess Biotechniq. 2015;5:e131. 